\title{
Electromechanical abdominal massage and colonic function in individuals with a spinal cord injury and chronic bowel problems
}

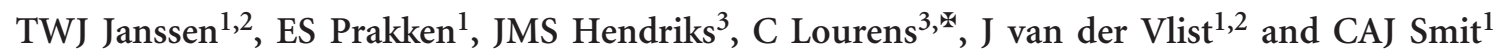

Study Design: A prospective intervention of noninvasive abdominal massage using an electromechanical apparatus on bowel function in individuals with spinal cord injury (SCl).

Objectives: To evaluate the effects of noninvasive abdominal massage using an electromechanical apparatus on bowel function in individuals with $\mathrm{SCl}$ and chronic bowel problems. This easy-to-use apparatus can be applied by the patients at home without the help of a therapist.

Setting: Homes of community-living individuals.

Methods: Twenty-one subjects with SCl were instructed to use the massage apparatus daily for 20 min during a 10 -week period. Compliance, effects, side effects and user satisfaction were assessed using questionnaires.

Results: Fifteen subjects completed the 10-week period. Although some characteristics of defecation changed positively for some of the subjects (time to result, amount, consistency), none felt better or more confident after using the massage device. In addition, some individuals experienced negative side effects (predominantly pain or discomfort). The overall satisfaction with the device is ambiguous, with half of the group judging the device as insufficient and the other half as at least adequate.

Conclusion: The use of an electromechanical massage device does not improve bowel function in most individuals with SCI who have chronic bowel problems. Why some subjects benefit and others do not should be investigated in future studies.

Spinal Cord (2014) 52, 693-696; doi:10.1038/sc.2014.101; published online 17 June 2014

\section{INTRODUCTION}

Constipation, gastrointestinal pain and fecal incontinence are common problems in individuals with a spinal cord injury (SCI). ${ }^{1-3}$ Also, both colon transit time, especially in the distal part of the colon, and the defecation process are considerably longer compared with healthy individuals, ${ }^{1,2,4-7}$ which can lead to complications during evacuation and consequently to an increased risk of developing a neurogenic colon. ${ }^{8}$ These problems have a marked impact on activities of daily living (for example, causing restrictions in diet and outdoor activities) and have a negative influence on quality of life in individuals with SCI. ${ }^{1,7}$

Regular procedures to assist colon evacuation are placing an enema, manual fecal evacuation, digital rectal stimulation and increased abdominal pressure. In addition, oral and/or rectal medication is often used to relieve gastrointestinal problems, as well as diet changes and surgical procedures (for example, a stoma). Despite these interventions, constipation-related symptoms are still highly prevalent in individuals with SCI. ${ }^{2}$

One of the factors that contribute to the constipation of individuals with SCI is their immobility, which has already been shown in other populations such as the frail elderly. ${ }^{9}$ A method that might counteract this immobility is abdominal massage, which has been stated as a potential effective method to reduce chronic constipation and other bowel problems. ${ }^{10}$ In addition, in a group of individuals with stroke significant improvement was found in the frequency of defecation and degree of constipation after abdominal meridian massage. ${ }^{11}$ Also, positive effects of abdominal massage on constipation symptoms in individuals with multiple sclerosis have been reported. ${ }^{12}$ However, Klauser et al. ${ }^{13}$ were not able to show a significant improvement of colonic function in healthy people and those with chronic constipation.

Currently little is known about the effect of abdominal massage in individuals with SCI. However, some (pilot) studies on manual abdominal massage $e^{14,15}$ as well as on neuromuscular stimulation of the abdominal wall ${ }^{16}$ have shown positive effects. Yet, these massage methods require a therapist or electrodes for application.

The purpose of the present study was to evaluate the effects of noninvasive abdominal massage using an electromechanical apparatus on bowel function in individuals with SCI and chronic bowel problems. This easy-to-use apparatus might be a great alternative for the time-consuming and expensive massages executed by therapists.

\section{MATERIALS AND METHODS}

Participants

Twenty-one individuals with chronic SCI ( 3 female, 18 male, age $56.5 \pm 11$ years, range 38-79 years, time since injury $221 \pm 146$ months; ASIA (American

${ }^{1}$ Amsterdam Rehabilitation Research Center I Reade, Amsterdam, The Netherlands; ${ }^{2}$ MOVE Research Institute Amsterdam, Faculty of Human Movement Sciences, VU University, Amsterdam, The Netherlands and ${ }^{3}$ Dutch Spinal Cord Injury Organization, Groningen, The Netherlands

Correspondence: Professor TWJ Janssen, Amsterdam Rehabilitation Research Center I Reade, Overtoom 283, Amsterdam 1054WP, The Netherlands.

E-mail: t.janssen@reade.nl

Deceased.

Received 6 January 2014; revised 2 April 2014; accepted 11 May 2014; published online 17 June 2014 
Spinal Injury Association) A $(N=8)$, ASIA B $(N=2)$, ASIA C-D $(N=11))$, recruited through mailings by the Dutch Spinal Cord Injury Organization, volunteered to participate in this study, approved by a local institutional review board. Thirteen subjects had cervical injury resulting in a tetraplegia and eight had a paraplegia. Eight subjects had a complete lesion. All subjects lived at home and indicated having chronic bowel problems. All participants signed an informed consent before taking part in the study. Average bowel movement rate was daily $(n=8)$, every other day $(n=5)$, every third day $(n=5)$, weekly $(n=1)$ or irregular $(n=2)$. Average time to defecation was less than $10 \mathrm{~min}$ $(n=4)$, between 10 and $15 \mathrm{~min}(n=5)$, between 16 and $20 \mathrm{~min}(n=2)$, between 21 and $25 \min (n=1)$ and more than $25 \min (n=9)$. All subjects regularly used laxative medication, such as Bisacodyl and Microlax. We certify that all applicable institutional and governmental regulations concerning the ethical use of human volunteers were followed during the course of this research.

\section{Study design}

During a 10-week period, participants used the massage apparatus daily for $20 \mathrm{~min}$. This self-applied therapy was incorporated in the daily-life situation, $6-10 \mathrm{~h}$ before the usual time of defecation. Compliance, effects, side effects and user satisfaction were assessed using questionnaires. Participants were instructed to keep diet and medication use stable during the treatment period.

\section{Massage device}

An electromechanical massage device (Free-Lax, ADM-Advanced Dialysis Methods Ltd., Bnei Brak, Israel) was used to massage the abdomen at the level of the transverse part of the colon, with the purpose of stimulating bowel function and improving peristaltic action. The apparatus consists of a slightly curved hard plastic box, which is positioned over the upper thighs of the seated participant with two small supports. A belt is tied around the user's back, allowing the apparatus to exert pressure on the abdomen with two disks. These disks massage the abdomen with a patented inward-outward oscillating movement pattern when the apparatus is switched on using the control panel (see Figure 1). For the present study, the frequency of movement was set at $1.5 \mathrm{~Hz}$ (which is the middle of three possible frequencies) for all subjects. Eleven subjects used the device independently, whereas the remaining ten received assistance. During an instruction session, subjects or their assistants (for example, spouse) were instructed on how to use the massage device after which they took the device home.

\section{Questionnaires and outcome measures}

Before starting treatment (baseline), participants completed a questionnaire on defecation habits, use of laxative medication and diet. The usually experienced stool consistency was indicated on a modified Bristol stool form scale:

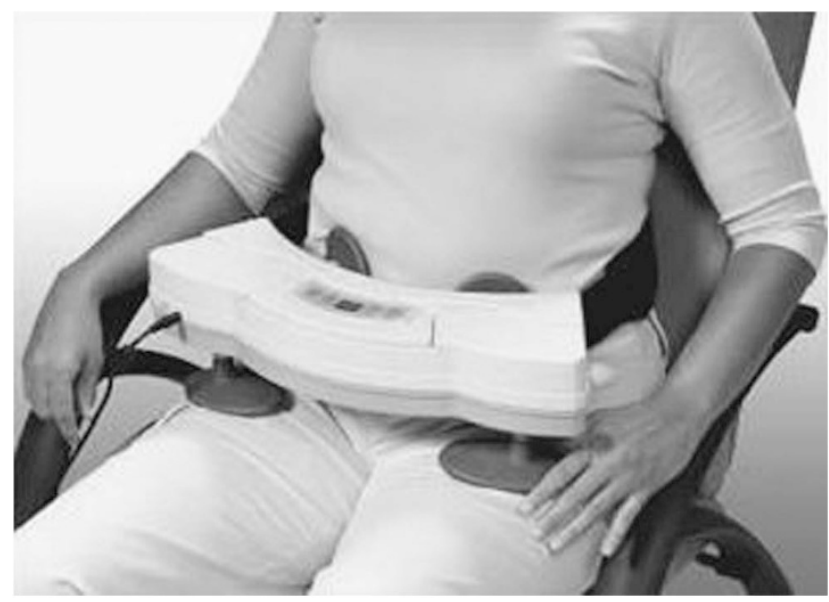

Figure 1 The Free-Lax massage device used by subjects with $\mathrm{SCl}$ in order to stimulate bowel function during the 10-week intervention period.
(1) separate hard lumps, like nuts (hard to pass), (2) sausage-shaped, (3) fluffy pieces with ragged edges, a mushy stool or (4) watery, no solid pieces. During the treatment period, a daily form on defecation details and a weekly form on therapy compliance and side effects were completed. The last questionnaire, concerning changes in defecation patterns since baseline, side effects and user subjective findings and satisfaction, was completed at the end of the 10-week period. The questions on defecation characteristics were: (1) Did the consistency of your stool change? (2) Did the shape of your stool change? (3) Did the amount of your stool change? (4) Did the time to result after starting the defecation procedure change? (5) Was there a change in incontinence episodes (no; yes, more often; yes, less often)? (6) Was there a change in flatulence (no; more; less)? The questions on subjective findings and satisfaction were: (1) What is the ease of use of the device? (2) Did you experience pain or discomfort while using the device? (3) Do you feel better after using the device for this period? (4) Do you feel more secure after using the device for this period? (5) Has the use of this device affected (positively or negatively) your daily life? (6) Would you like to continue using this device in the future? (7) What is your overall opinion about the device (very good, good, adequate, insufficient)? All forms were sent by email to the research assistant for subsequent analysis.

\section{Data analysis}

Descriptive statistics were used to evaluate the changes in defecation characteristics, the user satisfaction and the occurrence of side effects.

\section{RESULTS}

Not all subjects were able to complete the 10-week therapy. Two subjects had to stop the therapy because of medical reasons not related to the treatment (that is, pressure sores). More importantly, four subjects ended the program because the treatment resulted in abdominal pain or discomfort $(n=3)$, or unwanted change in stool consistency (too hard; $n=1$ ). The remaining 15 subjects completed the program and were included in the analyses.

Six subjects used the Free-Lax every day (70 days), nine subjects used it at least every second day ( $>35$ days) and six subjects used it less than half of the time ( $<35$ days), from which four used it less than 10 days. Reasons for not using the device every day varied from forgotten to medical reasons or pain/discomfort. About half of the subjects was able to put the Free-Lax on by their own, whereas the other half needed assistance. Although it was prescribed to use the apparatus seated, one subject found that too difficult and used it standing. All the subjects used the device 20 min every time, but one subject chose to use a higher frequency because the prescribed frequency showed no noticeable effect.

Tables 1 and 2 present the outcome of the questionnaire completed at the end of the 10-week period. Although a couple characteristics of defecation changed positively for some of the subjects (time to result, amount, consistency, Table 1), none of the 15 subjects who completed the 10-week period felt better or more confident after using the massage device. In addition, some subjects experienced pain or discomfort during the treatment (Table 2). Moreover, for the greater part of the subject group, the use of the apparatus had no effect on daily life and only three stated that they would continue using it despite the fact that the majority of the subjects found the device easy to use. The final conclusion is that the meanings are divided, with half of the group judging the device as insufficient and the other half as at least adequate.

\section{DISCUSSION}

The purpose of the present study was to evaluate whether electromechanical abdominal massage, supposedly counteracting the 
Table 1 Outcome questionnaire about changes in defecation and related characteristics at the end of the 10-week treatment period

\begin{tabular}{|c|c|}
\hline Question & Number of subjects \\
\hline \multicolumn{2}{|l|}{ Consistency } \\
\hline No change & 13 \\
\hline Softer & 2 \\
\hline Harder & 0 \\
\hline \multicolumn{2}{|l|}{ Shape } \\
\hline No change & 14 \\
\hline Sausage-shaped (from hard lumps) & 1 \\
\hline Diarrhea & 0 \\
\hline \multicolumn{2}{|l|}{ Amount } \\
\hline No change & 8 \\
\hline More & 3 \\
\hline Less & 1 \\
\hline Variable & 3 \\
\hline \multicolumn{2}{|l|}{ Time to result } \\
\hline No change & 6 \\
\hline Faster & 6 \\
\hline Variable & 3 \\
\hline \multicolumn{2}{|l|}{ Incontinence } \\
\hline No change & 15 \\
\hline More often & 0 \\
\hline Less often & 0 \\
\hline \multicolumn{2}{|l|}{ Flatulence } \\
\hline No change & 12 \\
\hline Less & 2 \\
\hline More & 1 \\
\hline
\end{tabular}

immobility, could improve bowel function in individuals with SCI having chronic bowel problems.

Although previous research in this field is scarce, a pilot study by Albers et al. ${ }^{14}$ in a small group of seven persons with SCI showed that abdominal massage in this group may be useful. After a week of daily specific non-mechanical (that is, manual) abdominal massage before breakfast some positive changes had occurred in the frequency and duration of defecation. Moreover, subjects indicated that they found the massage a comfortable intervention. This social aspect of the treatment was described in a study about patients' experiences of receiving abdominal massage. ${ }^{17}$ Subjects felt they were being touched physically and emotionally and that the massage helped them to manage living with constipation better. Nevertheless, also without this emotional aspect, massage has been found effective. For example, Korsten et al. ${ }^{16}$ used an abdominal band with implanted electrodes to improve the defecation process by inducing contractions of the abdominal muscles. They found a reduced time to defecation and total defecation time in a group of eight individuals with SCI. Hence, we expected electromechanical abdominal massage to improve the defecation process. The results of the present study, however, show that the used mechanical abdominal massage had no major effects in most of our subjects and even adverse effects in some subjects. A possible explanation for the lack of effect is that a massage device is not able to stimulate the bowel as effectively as a manual massage.
Table 2 Outcome questionnaire about the overall evaluation of and satisfaction with the Free-Lax at the end of the 10-week treatment period

\begin{tabular}{|c|c|}
\hline \multirow{2}{*}{\multicolumn{2}{|c|}{ Ease of use }} \\
\hline & \\
\hline Very good & 3 \\
\hline Good & 5 \\
\hline Adequate & 5 \\
\hline Insufficient & 2 \\
\hline \multicolumn{2}{|c|}{ Pain while using Free-Lax ${ }^{a}$} \\
\hline Yes & 5 \\
\hline No & 15 \\
\hline \multicolumn{2}{|l|}{ Feel better } \\
\hline Yes & 0 \\
\hline No & 15 \\
\hline \multicolumn{2}{|c|}{ Feel more secure } \\
\hline Yes & 0 \\
\hline No & 15 \\
\hline \multicolumn{2}{|c|}{ Effect on daily life } \\
\hline Positive & 3 \\
\hline Negative & 1 \\
\hline No & 10 \\
\hline Hardly & 1 \\
\hline \multicolumn{2}{|c|}{ Continue Free-Lax } \\
\hline Yes & 3 \\
\hline No & 6 \\
\hline Yes/no & 6 \\
\hline \multicolumn{2}{|c|}{ Final judgement } \\
\hline Very good & 2 \\
\hline Good & 2 \\
\hline Adequate & 4 \\
\hline Insufficient & 7 \\
\hline
\end{tabular}

aAlso subjects who did not complete the 10-week period, answered this question.

However, Mimidis et al. ${ }^{18}$ showed good effects in constipated elderly patients using the exact same massage device as in the present study. Moreover, they found no side effects. It is difficult to explain the differences in results between the study of Mimidis et al. and the present study. Clearly, the subject groups differ, with the elderly being inactive but not paralyzed. It is possible that the Free-Lax is capable to compensate for the negative effects of being inactive but not for that of paralysis. However, it is difficult to underpin this hypothesis as the mechanisms by which massage would improve bowel function are not entirely clear yet. It has been suggested that the massage-induced mechanical effect enhances mass movement of the gut and in this way resembles normal peristaltic contraction. ${ }^{19}$ However, recent evidence suggests that it is doubtful whether massage is able to manually force stool through the intestines. ${ }^{10}$ Another possible mechanism by which massage can diminish constipation is stimulating rectal muscle contraction by increasing the intra-abdominal pressure, ${ }^{20}$ which is low in individuals with SCI due to paralysis of the abdominal muscles. In addition, it is thought that stretch receptors are activated which can reinforce the gastrocolic reflex, causing an increase in motility of the gastrointestinal tract. ${ }^{21}$ Also activation of the parasympathetic nervous system, which is highly involved in 
digestion, may reduce constipation. If this is the case, it is very important that the subjects feel comfortable with the massage device. Otherwise, the sympathetic division of the nervous system, which is associated with stress, could negatively counteract the activation of the parasympathetic division. Although the subjects in the present study used the massage device in a trusted environment (at home), it is possible that they were not comfortable enough to benefit from activation of the parasympathetic nervous system. This might also explain that some subjects show beneficial effects of the massage and others do not as some might have been more comfortable than others. However, this cannot be concluded from the present results and should be investigated in future studies.

In conclusion, the results of this study show that the use of an electromechanical massage device does not improve bowel function in all individuals with SCI who have chronic bowel problems. In some individuals, the time to defecation was somewhat faster, but the majority of users did not notice any changes. In addition, in some individuals, this device may even cause side effects (predominantly pain and discomfort) that inhibit use of this device. As there is no homogeneous response to the device, it would be valuable to clarify the distinct characteristics of the individuals who benefit from the electromechanical abdominal massage. In this way, it would become possible to recommend the device to specific individuals. Further research is needed.

\section{Limitations}

A limitation of our study is that data were mainly based on subjective reports and diaries, which might be subjected to bias. We did not choose for a double-blind design. Also, we did not control for the actual use of the device. It is possible that some subjects used it more or less than the prescribed 20 min per day.

\section{DATA ARCHIVING}

There were no data to deposit.

\section{CONFLICT OF INTEREST}

The authors declare no conflict of interest.

\section{ACKNOWLEDGEMENTS}

We acknowledge the assistance of Marijke de Leeuw in collecting the questionnaires and of Andrea van London in preparing the manuscript. We are also thankful to Cedric Lourens, who was one of the initiators of this project, but who sadly passed away before the publication of this manuscript.
1 Han TR, Kim JH, Kwon BS. Chronic gastrointestinal problems and bowel dysfunction in patients with spinal cord injury. Spinal Cord 1998; 36: 485-490.

2 Harari D, Sarkarati M, Gurwitz JH, McGlinchey-Berroth G, Minaker KL. Constipationrelated symptoms and bowel program concerning individuals with spinal cord injury. Spinal Cord 1997; 35: 394-401.

3 Menter R, Weitzenkamp D, Cooper D, Bingley J, Charlifue S, Whiteneck G. Bowel management outcomes in individuals with long-term spinal cord injuries. Spinal Cord 1997; 35: 608-612.

4 Fajardo NR, Pasiliao RV, Modeste-Duncan R, Creasey G, Bauman WA, Korsten MA Decreased colonic motility in persons with chronic spinal cord injury. Am J Gastroenterol 2003; 98: 128-134.

5 Glickman S, Kamm MA. Bowel dysfunction in spinal-cord-injury patients. Lance 1996; 347: 1651-1653.

6 Keshavarzian A, Barnes WE, Bruninga K, Nemchausky B, Mermall H, Bushnell D. Delayed colonic transit in spinal cord-injured patients measured by indium-111 Amberlite scintigraphy. Am J Gastroenterol 1995; 90: 1295-1300.

7 Lynch AC, Wong C, Anthony A, Dobbs BR, Frizelle FA. Bowel dysfunction following spinal cord injury: a description of bowel function in a spinal cord-injured population and comparison with age and gender matched controls. Spinal Cord 2000; 38 717-723.

8 Stiens SA, Bergman SB, Formal CS. Spinal cord injury rehabilitation. 4. Individual experience, personal adaptation, and social perspectives. Arch Phys Med Rehabil 1997; 78: S65-S72.

9 Melkersson M, Andersson $\mathrm{H}$, Bosaeus I, Falkheden $\mathrm{T}$. Intestinal transit time in constipated and non-constipated geriatric patients. Scand J Gastroenterol 1983; 18: 593-597.

10 Sinclair M. The use of abdominal massage to treat chronic constipation. J Bodyw Mov Ther 2011; 15: 436-445.

11 Jeon SY, Jung HM. [The effects of abdominal meridian massage on constipation among CVA patients]. Taehan Kanho Hakhoe Chi 2005; 35: 135-142.

12 McClurg D, Hagen S, Hawkins S, Lowe-Strong A. Abdominal massage for the alleviation of constipation symptoms in people with multiple sclerosis: a randomized controlled feasibility study. Mult Scler 2011; 17: 223-233.

13 Klauser AG, Flaschentrager J, Gehrke A, Muller-Lissner SA. Abdominal wall massage: effect on colonic function in healthy volunteers and in patients with chronic constipation. Z Gastroenterol 1992; 30: 247-251.

14 Albers B, Cramer H, Fischer A, Meissner A, Schurenberg A, Bartholomeyczik S. [Abdominal massage as intervention for patients with paraplegia caused by spinal cord injury-a pilot study]. Pflege Z 2006; 59: 2-8.

15 Ayas S, Leblebici B, Sozay S, Bayramoglu M, Niron EA. The effect of abdominal massage on bowel function in patients with spinal cord injury. Am J Phys Med Rehabil 2006: 85: 951-955.

16 Korsten MA, Fajardo NR, Rosman AS, Creasey GH, Spungen AM, Bauman WA Difficulty with evacuation after spinal cord injury: colonic motility during sleep and effects of abdominal wall stimulation. J Rehabil Res Dev 2004; 41: 95-100.

17 Lamas K, Graneheim UH, Jacobsson C. Experiences of abdominal massage for constipation. J Clin Nurs 2012; 21: 757-765.

18 Mimidis K, Galinsky D, Rimon E, Papadopoulos V, Zicherman Y, Oreopoulos D. Use of a device that applies external kneading-like force on the abdomen for treatment of constipation. World J Gastroenterol 2005; 11: 1971-1975.

19 Emly MC, Vail, A S. Colonic motility in profoundly disabled people: a comparison of massage and laxative therapy in the management of constipation. Physiotherapy 1998; 84: 6 .

20 Liu Z, Sakakibara R, Odaka T, Uchiyama T, Yamamoto T, Ito T et al. Mechanism of abdominal massage for difficult defecation in a patient with myelopathy (HAM/TSP). I Neurol 2005; 252: 1280-1282.

21 Brookes SJ, Chen BN, Costa M, Humphreys CM. Initiation of peristalsis by circumferential stretch of flat sheets of guinea-pig ileum. J Physiol 1999; 516 525-538. 